\title{
Desempenho agronômico de genótipos de amendoim nas condições de Santo Antônio do Leste-MT
}

Submetido - 12 jul. $2020 \quad$ Aprovado - 07 ago. $2020 \quad$ Publicado - 14 out. 2020
dol http://dx.doi.org/10.17648/sas.v1i1.21

Fabiano de Souza Agulhon (D)

Agulhon Agroindustrial Ltda., Santo Antônio do Leste,MT, fabianoagulhon@yahoo.com.br.

Jair Heuert (D)

Programa de Melhoramento do Amendoim - Embrapa, Santo Antônio de Goiás, GO, jair.heuert@embrapa.br.

Programa de Melhoramento do -Amendoim kennedybrunno22@gmail.com.

- Embrapa, Santo Antônio de Goiás, GO,

Maxuel Fellipe Nunes Xavier (iD)

Discente de Agronomia do IFMT Campus São Vicente - Centro de Referência de Campo Verde, Campo Verde, MT, maxuelfellipe90@gmail.com.

Taís de Moraes Falleiro Suassuna iD

Programa de Melhoramento do Amendoim - Embrapa, Santo Antônio de Goiás, GO, tais.suassuna@embrapa.br.

\section{RESUMO}

Objetivou-se avaliar desempenho agronômico de genótipos de amendoim nas condições de Santo Antônio do Leste-MT. O experimento foi instalado em área de culturas anuais na Fazenda Santo Antônio, localizada no município de Santo Antônio do Leste-MT, com altitude média de 620 metros, na safra 2019/20, utilizando o sistema de cultivo convencional, com semeadura realizada no dia 30 de outubro de 2019. O delineamento experimental utilizado foi em blocos casualizados, com quatro repetições e nove tratamentos, sendo eles seis linhagens: 17-1253 OL, 18-1952 OL, 18-1973 OL, 18-2091 OL, 18-2133 OL, 18-2136 OL e três cultivares: BRS 421 OL, BRS 423 OL e BRS 425 OL desenvolvidas pelo Programa de Melhoramento do Amendoim da Embrapa. As parcelas foram constituídas por duas linhas de três metros de comprimento, com intervalo de três metros entre parcelas, espaçamentos entre linhas de noventa centímetros. 0 estande médio estabelecido foi de 15 plantas por metro linear. Aos 130 dias após a semeadura (DAE), foi realizada a inversão mecanizada das plantas, e avaliado massa de 100 grãos $(g)$ e produtividade de vagens (kg ha-1). As linhagens 18-1973 OL, 18-2091 OL, 17-1253 OL e 18-2133 $O L$ foram as mais produtivas nas condições edafoclimáticas do Cerrado Mato-Grossense, com produtividades superiores a $6.300 \mathrm{~kg} \mathrm{ha}^{-1}$.

Palavras-chave: Arachis hypogaea L.; Programa de Melhoramento; Produtividade; Amendoim em Mato Grosso.

\section{Agronomic performance of peanut genotypes under the conditions of Santo Antônio do Leste-MT}

\section{ABSTRACT}

The objective was to evaluate the agronomic performance of peanut genotypes under the conditions of Santo Antônio do Leste-MT. The experiment was installed in an annual crop area at Fazenda Santo Antônio, located in the municipality of Santo Antônio do Leste-MT, with an average altitude of 620 meters, in the 2019/20 harvest, using the conventional cultivation 
system, with sowing carried out on the day October 30, 2019. The experimental design used was in randomized blocks, with four replications and nine treatments, including six lines: 171253 OL, 18-1952 OL, 18-1973 OL, 18-2091 OL, 18-2133 OL, 18-2136 OL and three cultivars: BRS 421 OL, BRS 423 OL and BRS 425 OL developed by Embrapa's Peanut Breeding Program. The plots consisted of two lines three meters long, with an interval of three meters between plots, with spacing between lines of ninety centimeters. The average stand established was 15 plants per linear meter. At 130 days after sowing (DAE), mechanized inversion of the plants was carried out, and a mass of 100 grains $(\mathrm{g})$ and pod yield $\left(\mathrm{kg} \mathrm{ha}^{-1}\right)$ were evaluated. The strains 18$1973 \mathrm{OL}, 18-2091 \mathrm{OL}, 17-1253 \mathrm{OL}$ and 18-2133 OL were the most productive in the edaphoclimatic conditions of Cerrado Mato-Grossense, with yields above 6,300 kg ha-1.

Keywords: Arachis hypogaea L.; Improvement Program; Productivity; Peanut in Mato Grosso.

\title{
Rendimiento agronómico de los genotipos de maní en las condiciones de Santo Antônio do Leste-MT
}

\section{RESUMEN}

\begin{abstract}
El objetivo fue evaluar el rendimiento agronómico de los genotipos de maní en las condiciones de Santo Antônio do Leste-MT. El experimento se instaló en un área de cultivo anual en Fazenda Santo Antônio, ubicado en el municipio de Santo Antônio do Leste-MT, con una altitud promedio de 620 metros, en la cosecha 2019/20, utilizando el sistema de cultivo convencional, con la siembra realizada el día 30 de octubre de 2019. El diseño experimental utilizado fue en bloques aleatorios, con cuatro repeticiones y nueve tratamientos, incluidas seis líneas: 17-1253 OL, 18-1952 OL, 18-1973 OL, 18-2091 OL, 18-2133 OL, 18-2136 OL y tres cultivares: BRS 421 OL, BRS 423 OL y BRS 425 OL desarrolladas por el Programa de Mejoramiento de Maní de Embrapa. Las parcelas constaban de dos líneas de tres metros de largo, con un intervalo de tres metros entre parcelas, con un espacio entre líneas de noventa centímetros. El stand promedio establecido fue de 15 plantas por metro lineal. A los 130 días después de la siembra (DAE), se realizó una inversión mecanizada de las plantas, y se evaluó una masa de 100 granos (g) y rendimiento de vaina $\left(\mathrm{kg} \mathrm{ha}^{-1}\right)$. Las cepas 18-1973 OL, 18-2091 OL, 17-1253 OL y 18-2133 OL fueron las más productivas en las condiciones edafoclimáticas de Cerrado Mato-Grossense, con rendimientos superiores a $6.300 \mathrm{~kg} \mathrm{ha}^{-1}$.
\end{abstract}

Palabras clave: Arachis hypogaea L.; Programa de Mejoramiento; Productividad; Maní em Mato Grosso.

\section{Introdução}

O amendoim (Arachis hypogaea L.) adequa-se ao sistema de rotação de culturas, pois é cultura anual e apresenta benefícios ao solo por ser leguminosa. Está cultura disponibiliza nitrogênio que possibilita a melhoria da fertilidade do solo, advindo da fixação biológica de nitrogênio (SILVA, 2020). O amendoim atua na ciclagem de nutrientes do sistema, pois absorve nutrientes das camadas mais profundas do perfil do solo, trazendoas de volta à superfície. Depois que o amendoim é colhido, o resíduo vegetal que fica no solo se decompõe e fornece nutrientes para os cultivos subsequentes (PERES, 2003). Com isso, a cultura do amendoim pode ser 
inserida na rotação de culturas, em primeira safra, podendo influenciar na descompactação do solo, auxiliar o controle de plantas daninhas por utilizar herbicidas com diferentes mecanismos de ação, e pode ser uma alternativa economicamente viável.

O munícipio de Santo Antônio do Leste-MT possui destaque pelas altas produtividades de algodão, milho e soja. Um sistema definido com cultivo de soja na safra e milho segunda safra ou soja na safra e algodão segunda safra. Segundo Tubbs (2015), sistemas alternativos de cultivo de amendoim devem ser pesquisados, pois a cultura pode ser capaz de manter rendimentos e lucros maximizados, com incidência e custos minimizados de pragas para os cultivos subsequentes. Além de ser resistente e/ou não hospedeira de nematoides.

Os nematoides se encontram entre as principais pragas na agricultura atualmente, estima-se que os nematoides parasitos de plantas consomem aproximadamente $10 \%$ da produção agrícola global, ocasionando em perdas econômicas anuais avaliadas em mais de U\$125 bilhões. No Brasil, os nematoides são um dos problemas fitossanitários mais importantes nos cultivos. Estudos indicam que 0 amendoim pode ser efetivo na redução populacional das espécies de $M$. incognita, $R$. reniformis (MENTEN, 2018), M. javanica, H. glycines (MIRANDA; MIRANDA, 2018) e P. brachyurus (MENDES et al., 2019; AMBROSANO et al., 2011). Objetivouse avaliar desempenho agronômico de genótipos de amendoim nas condições de Santo Antônio do Leste, sendo seis linhagens e três cultivares.

\section{Material e métodos}

O experimento foi instalado em área de culturas anuais na Fazenda Santo Antônio ( $14^{\circ} 44^{\prime} 36^{\prime \prime} S$ e $53^{\circ} 23^{\prime} 36^{\prime \prime}$ W), localizada no município de Santo Antônio do Leste-MT, com altitude média de 620 metros, na safra 2019/20, utilizando o sistema de cultivo convencional, com semeadura realizada no dia 30 de outubro de 2019. De acordo com a classificação Köppen-Geiger, o clima da região é do tipo Aw (clima tropical com estação seca de inverno). 
O delineamento experimental utilizado foi em blocos casualizados, com quatro repetições e nove tratamentos, sendo eles seis linhagens: 171253 OL, 18-1952 OL, 18-1973 OL, 18-2091 OL, 18-2133 OL, 18-2136 OL e três cultivares: BRS 421 OL, BRS 423 OL e BRS 425 OL desenvolvidas pelo Programa de Melhoramento do Amendoim da Embrapa. As parcelas foram constituídas por duas linhas de três metros de comprimento, com intervalo de três metros entre parcelas, espaçamentos entre linhas de noventa centímetros.

Foi realizado o manejo fitossanitário e cultural conforme as recomendações para a cultura. $O$ estande médio estabelecido foi de 15 plantas por metro linear. Aos 130 dias após a semeadura (DAE), foi realizada a inversão mecanizada das plantas. Após cinco dias de secagem na área experimental, foram feitas as avaliações de massa de 100 grãos (g), rendimento (\%) e produtividade de vagens $\left(\mathrm{kg} \mathrm{ha}^{-1}\right.$ e sacas alqueire $\left.{ }^{-1}\right)$.

A avaliação rendimento (\%) consistiu na retirada de $2,0 \mathrm{~kg}$ de amendoim em vagens de cada parcela sendo considerado 100\%. Após o beneficiamento mecanizado simulando um sistema industrial foi feito a massa de grãos e a casca foi desprezada, com isso calculando a porcentagem de rendimento das parcelas.

Os dados obtidos foram submetidos à análise de variância (Teste $F$ ) e as médias dos tratamentos foram comparados pelo teste de Scott-Knott a $5 \%$ de probabilidade, por meio do programa computacional SISVAR 5.6 (FERREIRA, 2019).

\section{Resultados e discussão}

As linhagens e cultivares de amendoim apresentaram diferença significativa para a massa de 100 grãos (g), rendimento (\%) e produtividade de vagens $\left(\mathrm{kg} \mathrm{ha}^{-1}\right)$ (Tabela 1). As maiores massas de 100 grãos foram obtidas nos genótipos BRS $421 \mathrm{OL}(81,6 \mathrm{~g}), 17-1253 \mathrm{OL}(81,0 \mathrm{~g}), 18-1973$ OL (80,7 g) e 18-2133 OL (78,4 g). Os genótipos 18-1952 OL (76,6 g) BRS $425 \mathrm{OL}(75,9 \mathrm{~g})$ e BRS $423 \mathrm{OL}(75,6 \mathrm{~g})$ obtiveram massas intermediárias. As menores foram obtidas com as linhagens $18-2136 \mathrm{OL}(74,1 \mathrm{~g})$ e 18-2091 OL 
(71,1 g). Corroborando Santin et al. (2019), observaram massa de 100 grãos de 79,3 g com a cultivar BRS 421 OL em Campo Verde-MT. Martins et al. (2019), obtiveram massas semelhantes com as cultivares BRS $425 \mathrm{OL}(74,0$ g), BRS $423 \mathrm{OL}(75,9 \mathrm{~g})$ e as linhagens $18-1952 \mathrm{OL}(78,4 \mathrm{~g})$ e $18-2136 \mathrm{OL}$ (73,9 g) em Santo Antônio de Goiás-GO.

Tabela 1. Massa de 100 grãos (g), rendimento (\%) e produtividade de vagens ( $\mathrm{kg} \mathrm{ha}^{-1}$ e sacas alqueire ${ }^{-1}$ ) em função de diferentes genótipos de amendoim. Santo Antônio do Leste-MT, 2019/20.

\begin{tabular}{|c|c|c|c|c|}
\hline \multirow{2}{*}{ Genótipos } & \multirow{2}{*}{$\begin{array}{c}\text { Massa de } \\
100 \text { grãos } \\
\text { (g) }\end{array}$} & \multirow{2}{*}{$\begin{array}{c}\text { Rendimento } \\
(\%)\end{array}$} & \multicolumn{2}{|c|}{ Produtividade de vagens } \\
\hline & & & $\left(\mathrm{kg} \mathrm{ha}^{-1}\right)$ & (sacas alqueire $^{-1}$ ) \\
\hline $18-1973 \mathrm{OL}$ & $80,7 \mathrm{a}$ & $79,1 \mathrm{a}$ & $6.880,3 \mathrm{a}$ & 666,0 \\
\hline $18-2091 \mathrm{OL}$ & $71,1 \mathrm{e}$ & $78,1 \mathrm{a}$ & $6.777,4 \mathrm{a}$ & 656,1 \\
\hline $17-1253 \mathrm{OL}$ & $81,0 \mathrm{a}$ & $78,5 \mathrm{a}$ & $6.605,0 \mathrm{a}$ & 639,4 \\
\hline $18-2133 \mathrm{OL}$ & $78,4 b$ & $71,1 d$ & $6.315,0 \mathrm{a}$ & 611,3 \\
\hline BRS 425 OL & $75,9 \mathrm{c}$ & $74,5 \mathrm{c}$ & $5.955,1 \mathrm{~b}$ & 576,5 \\
\hline BRS $423 \mathrm{OL}$ & $75,6 \mathrm{c}$ & $75,6 \mathrm{~b}$ & $5.759,8 \mathrm{~b}$ & 557,5 \\
\hline BRS 421 OL & $81,6 \mathrm{a}$ & $72,7 d$ & $5.754,1 \mathrm{~b}$ & 557,0 \\
\hline $18-1952 \mathrm{OL}$ & $76,6 \mathrm{c}$ & $77,1 \mathrm{~b}$ & $5.608,2 \mathrm{~b}$ & 542,9 \\
\hline $18-2136 \mathrm{OL}$ & $74,1 \mathrm{~d}$ & $73,3 \mathrm{c}$ & $5.274,1 \mathrm{~b}$ & 510,5 \\
\hline Média & 77,2 & 75,6 & $6.103,2$ & 590,8 \\
\hline C.V. (\%) & 1,1 & 1,9 & 9,3 & - \\
\hline $\operatorname{Pr}>\mathrm{Fc}$ & $>0,0001^{*}$ & $>0,0001^{*}$ & $0,0034^{*}$ & - \\
\hline
\end{tabular}

A média de rendimento do experimento foi $75,6 \%$. Os genótipos 18 1973 OL (79,1\%), 17-1253 OL (78,5\%), 18-2091 OL (78,1\%), 18-1952 OL $(77,1 \%)$ e BRS $423 \mathrm{OL}(75,6 \%)$ obtiveram os maiores rendimentos e mantiveram-se acima da média experimental. Os menores rendimentos e que são inferiores à média experimental, foram obtidos nos genótipos BRS 425 OL (74,5\%), 18-2136 OL (73,3\%), BRS 421 OL (72,7\%) e 18-2133 OL $(71,1 \%)$.

Observa-se as linhagens $18-1973 \mathrm{OL}\left(6.880,3 \mathrm{~kg} \mathrm{ha}^{-1}\right), 18-2091 \mathrm{OL}$ (6.777,4 kg ha-1), 17-1253 OL (6.605,0 $\left.\mathrm{kg} \mathrm{ha}^{-1}\right)$ e $18-2133 \mathrm{OL}\left(6.315,0 \mathrm{~kg} \mathrm{ha}^{-}\right.$ 1) com as maiores produtividades, quando comparadas aos demais genótipos. As cultivares BRS $425 \mathrm{OL}\left(5.955,1 \mathrm{~kg} \mathrm{ha}^{-1}\right)$, BRS $423 \mathrm{OL}(5.759,8$ $\left.\mathrm{kg} \mathrm{ha}^{-1}\right)$, BRS $421 \mathrm{OL}\left(5.754,1 \mathrm{~kg} \mathrm{ha}^{-1}\right)$ e as linhagens $18-1952 \mathrm{OL}(5.608,2$ 
$\left.\mathrm{kg} \mathrm{ha}{ }^{-1}\right)$ e 18-2136 OL (5.274,1 $\left.\mathrm{kg} \mathrm{ha}^{-1}\right)$ obtiveram as menores produtividades.

Constata-se que mesmo sem diferirem entre si, as linhagens 181952 OL e 18-2136 OL produziram menos que as cultivares BRS 425 OL, BRS $423 \mathrm{OL}$ e BRS $421 \mathrm{OL}$. No entanto, todos os genótipos apresentaram produtividades superiores a $5.200 \mathrm{~kg} \mathrm{ha}^{-1}$, ou seja, produziram cerca de $46 \%$ acima da estimativa média nacional (3.554 $\left.\mathrm{kg} \mathrm{ha}^{-1}\right)$ da CONAB (2020).

Corroborando ao presente estudo, Martins et al. (2019), observaram resultados semelhantes com as linhagens $17-1253 \mathrm{OL}\left(6.735,6 \mathrm{~kg} \mathrm{ha}^{-1}\right)$ e 18-2133 OL (6.531,6 kg ha-1) nas condições de Santo Antônio de Goiás-GO. Rizzi et al. (2019) obtiveram produtividades de $5.840,3 \mathrm{~kg} \mathrm{ha}^{-1}$ e $5.641,7 \mathrm{~kg}$ $\mathrm{ha}^{-1}$ com as cultivares BRS $421 \mathrm{OL}$ e BRS $423 \mathrm{OL}$, respectivamente, no município de Sorriso-MT. Santin et al. (2019), observaram produtividade de $5.947,9 \mathrm{~kg} \mathrm{ha}^{-1}$ em Campo Verde-MT.

\section{Conclusões}

As linhagens 18-1973 OL, 18-2091 OL, 17-1253 OL e 18-2133 OL foram as mais produtivas nas condições edafoclimáticas do Cerrado MatoGrossense, com produtividades superiores a $6.300 \mathrm{~kg} \mathrm{ha}^{-1}$.

\section{Agradecimentos}

Os autores agradecem a Agulhon Agroindustrial e a Bokada Alimentos pela cooperação técnica e financeira que viabilizou a execução deste estudo. E a equipe da Fazenda Santo Antônio que prontamente realizaram a condução do experimento. Este experimento faz parte do Programa de Melhoramento do Amendoim da Embrapa, vinculada ao projeto SEG 20.18.01.021.00. 


\section{Referências}

AMBROSANO, E. J.; CANTARELLA, H.; AMBROSANO, G. M. B.; SHAMMASS, E. A.; DIAS, F. L. F.; ROSSI, F.; TRIVELIN, P. C. O.; MURAOKA, T.; SACHSM, R. C. C.; AZCÓN, R. Produtividade de cana-deaçúcar após cultivos de leguminosas. Bragantia, v. 70, n. 4, p. 810-818, 2011.

CONAB. Acompanhamento da Safra Brasileira de grãos. Safra 2019/20 Nono levantamento, v. 7, n. 9, p. 1-66, jun. 2020. Disponível em: <https://www.conab.gov.br/info-agro/safras>. Acesso em: 28 jun. 2020.

FERREIRA, D. F. SISVAR: a computer analysis system to fixed effects split plot type designs. Revista Brasileira de Biometria, [S.L.], v. 37, n. 4, p. 529, 20 dez. 2019. http://dx.doi.org/10.28951/rbb.v37i4.450.

Köppen-Geiger. Classificação climática de Köppen-Geiger. Disponível em: <https://portais.ufg.br/up/68/o/Classifica__o_Clim_tica_Koppen.pdf>. Acesso em: 29 jun. 2020.

MARTINS, K. B. B.; RODRIGUES, L. L.; HEUERT, J.; XAVIER, M. F. N.; SUASSUNA, T. M. F.; BETIOL, R. A. B. Desempenho agronômico de novas linhagens de amendoim no Cerrado. In: Anais do Encontro Sobre a Cultura do Amendoim, 16., 2019, Jaboticabal. Anais eletrônicos... Campinas: GALOÁ, 2019. Disponível em: <https://proceedings.science/encontroamendoim-2019/papers/desempenho-agronomico-de-novas-linhagens-deamendoim-no-cerrado>. Acesso em: 28 jun. 2020.

MENDES, A. C. S.; FREIRE, L. L.; PUERARI, H. H.; HEUERT, J.; ROCHA, M. R. Hospedabilidade de cultivar de amendoim a Pratylenchus brachyurus. In: Anais do Congresso Brasileiro de Nematologia, 36., 2019, Caldas Novas.

Resumos... Caldas Novas: CBN, 2019. Disponível em: <http://www.infobibos.com/anais/cbn/36/Resumos/Resumo36CBN_0168.pdf >. Acesso em 28 jun. 2020.

MENTEN, J. O. Nematoides: importância e manejo. 2018. Disponível em: $<$ https://www.grupocultivar.com.br/artigos/nematoides-importancia-emanejo>. Acesso em: 26 jun. 2020.

MIRANDA, L. L. D.; MIRANDA, L. D. Nematoides. 2018. Disponível em: $<$ http://www.nematoides.com.br/Content/Fotos/3JUL-

CartilhaNemat\%C3\%B3ides-atualizada.compressed.pdf>. Acesso em: 28 jun. 2020.

RIZZI, T. S.; OLIBONE, D.; LODEA, L.; HEUERT, J.; XAVIER, M. F. N.; SUASSUNA, T. M. F. Desempenho de cultivares de amendoim na região Médio-Norte Mato-Grossense. In: Anais do Encontro Sobre a Cultura do Amendoim, 16., 2019, Jaboticabal. Anais eletrônicos... Campinas: GALOÁ, 2019. Disponível em: <https://proceedings.science/encontro-amendoim- 
2019/papers/desempenho-de-cultivares-de-amendoim-na-regiao-medionorte-mato-grossense>. Acesso em: 28 jun. 2020.

SANTIN, V.; PEROZINI, A. C.; ARAÚJO, C.; GIRON, F. G.; HEUERT, J.; XAVIER, M. F. N.; SUASSUNA, T. M. F. Desempenho de cultivares de amendoim nas condições de Campo Verde-MT. In: Anais do Encontro Sobre a Cultura do Amendoim, 16., 2019, Jaboticabal. Anais eletrônicos... Campinas: GALOÁ, 2019. Disponível em: $<$ https://proceedings.science/encontro-amendoim-2019/papers/desempenhode-cultivares-de-amendoim-nas-condicoes-de-campo-verde-mt>. Acesso em: 28 jun. 2020.

SILVA, R. P. Amendoim brasileiro pode ter safra recorde este ano. 2020. Disponível em: <https://www.biodieselbr.com/noticias/materiaprima/outras/amendoim-brasileiro-pode-ter-safra-recorde-este-ano-160320>. Acesso em: 28 jun. 2020.

TUBBS, R. Sistemas de colheita e práticas agronômicas para rotações de amendoim. 2015.2 Disponível em: $<$ https://reeis.usda.gov/web/crisprojectpages/0226251-cropping-systemsand-agronomic-practices-for-peanut-rotations.html>. Acesso em: 26 jun. 2020. 